\title{
Thermodynamic and Conformational Properties of Styrene-Methyl Methacrylate Block Copolymers in Dilute Solution. III. Viscometric Behavior in Relation to Incompatibility of Parent Homopolymers
}

\author{
Hiroshi Ohnuma, ${ }^{*}$ Tadao Kotaka, ${ }^{* *}$ and Hiroshi Inagaki \\ Institute for Chemical Research, Kyoto University, \\ Uji, Kyoto 611, Japan.
}

(Received August 12, 1970)

\begin{abstract}
Viscometric behavior of nearly equimolar block copolymers of the poly(methyl methacrylate)-polystyrene-poly(methacrylate) type are studied in various solvents. The behavior is discussed in relation to the incompatibility of the parent homopolymers in each solvent. A correlation is found between the excluded-volume interactions in the block copolymer chain and the incompatibility of the homopolymer pair. A high compatibility is observed for the pair in cyclohexanol, and this reinforces our previous argument that this solvent may provide a genuine $\theta$ condition for the block copolymer, wherein random-flight statistics prevails. In certain common solvents, the homopolymer pair is fairly compatible, and the viscometric behavior of the block copolymer shows no anomalies: the so-called phenomenon of intrachain phase separation is unlikely to be taking place in such solvents. In $\theta$ solvents for either one of the homopolymers, they become less compatible. Nevertheless in a $\theta$ solvent for polystyrene the block copolymer shows no anomalies. On the other hand, in $\theta$ solvents for poly(methyl methacrylate), significant anomalies are observed. The anomalies must be due to the triblock architecture of the block copolymer chains: the two poly(methyl methacrylate) side-chains undergo intrachain association, thereby restricting allowable conformations of the central polystyrene chain.
\end{abstract}

KEY WORDS Block Copolymer / Styrene / Methyl Methacrylate / Triblock Chain/Ternary System/Intrinsic Viscosity/Incompatibility/ Long-Range Interaction/Intrachain Phase Separation/Conformational Anomaly/

In previous articles ${ }^{1-3}$ of this series we described the preparation and characterization of syrene (ST)-methyl methacrylate (MMA) block copolymers, ${ }^{2,4}$ and also their behavior in $\theta$ solvents ${ }^{1,3,4}$ as well as in good solvents. ${ }^{3,4}$ Generally block copolymers in dilute solution exhibit characteristic behavior resulting from thermodynamic interactions between chemically different blocks, each being a long stretch of monomers of a single type. Consequently the nature of such interactions should be, in some respects, similar to the interactions between parent homopolymers in the same solvent. The situation is in contrast to solutions of statistical

* Present Address: Department of Physics, Faculty of Science, Nagoya University, Chikusa-ku, Nagoya 464, Japan.

** To whom correspondence should be addressed. and alternating copolymers, wherein chemically different monomers are distributed along the chain with highly alternating sequences. ${ }^{5,6}$

It had been known that the incompatibility of two different polymers in the presence, as well as in the absence, of diluent is a general rule rather than an exception. ${ }^{7-9}$ This fact had led to an idea that a block copolymer composed of an incompatible homopolymer pair would exhibit a phenomenon of intrachain phase separation even in a common solvent toward both parent homopolymers. ${ }^{10,11}$ Many authors had attempted to interpret certain anomalies found in block copolymer solutions as the result of this phenomenon. ${ }^{10,11}$ In fact the phenomenon becomes apparent for certain block copolymers in bulk phase. ${ }^{12}$ However, in view of the compatibilizing nature of solvent in a ternary system 
involving two different polymers, ${ }^{8}$ we doubt whether the phenomenon would always take place in block copolymer chains in infinite dilution. We rather expect that the phenomenon should be influenced by solute molecular weight; comonomer composition and chain architecture, ${ }^{13}$ the extent of dilution and also, to some extent, the nature of the solvent.

To make these points clear, we examined the dilute solution behavior of ST-MMA block copolymers in correlation with the incompatibility of ternary systems involving polystyrene (PST), poly(methyl methacrylate) (PMMA), and the solvent.

\section{EXPERIMENTAL}

\section{Materials}

Block copolymer samples coded as series B are linear chains of PMMA-PST-PMMA type prepared by an anionic polymerization technique $^{15,15}$ at $-78^{\circ} \mathrm{C}$ with sodium biphenyl in tetrahydrofuran. ${ }^{100}$ The procedures were described elsewhere ${ }^{2}$ in greater detail, and will not be recounted here. We simply mention here the following two points. First, in each preparation an aliquot of precursor polystyryl carbanions was recovered and later used as a homo-PST sample (coded as series $\mathrm{H}$ ). The sample should have identical characteristics, i.e., the molecular weight and its distribution, microtacticity, etc., to the central PST-subchain of the product block copolymer. Secondly, precautions were taken to avoid any hazardous grafting reaction of the polystyryl carbanions on the ester groups of the PMMA-subchains by using a procedure of Freyss, Rempp, and Benoit. ${ }^{16}$ Each sample should be free from branched chains which often complicate the solution behavior of the sample. We also prepared a few homo-PMMA samples (coded as series $\mathbf{M}$ ) by the same anionic polymerization technique. ${ }^{2}$ The samples possess typically atatic configurations ${ }^{17}$ to which the microtacticity of PMMAsubchains of the block copolymer samples may be expected to be identical. Various organic solvents were carefully purified according to standard procedures appropriate to each. ${ }^{18}$

\section{Measurements}

Comonomer composition of each block co- polymer sample was determined by semimicrocombustion analysis. Measurements of osmotic pressure were made on a Mechrolab high speed membrane osmometer model 502. Measurements of light scattering were made on a Shimadzu light-scattering photometer of the modified Brice type. ${ }^{19,20}$ As an auxiliary technique for determining molecular weights, the sedimentation equilibrium method was employed. Molecular weight distributions of some homopolymer samples were determined by the sedimentation velocity method. The ultracentrifugation experiments were made on a Hitachi analytical ultracentrifuge model UCA-1A equipped with a Rayleigh interference and schlieren optics. ${ }^{21,22}$ Measurements of viscosities were made with Ubbelohde dilution viscometers. All these measurements were carried out according to our laboratory routines. The details are reported elsewhere, ${ }^{2,3,19-22}$ and will not be recounted here.

Maximum miscibility concentrations in ternary systems, PST : PMMA : solvent, were determined by a phase separation test. In each test, accurately weighed amounts of PST and PMMA (usually $100-\mathrm{mg}$ total polymers) were mixed with a solvent to be tested in a graduated tube with a ground glass stopper. The total amount was weighed accurately. The mixture was shaken vigorously at a somewhat elevated temperature. Then it was allowed to stand overnight in a thermostated bath (at $30^{\circ} \mathrm{C}$ unless otherwise noted). When the phase separation had taken place, the tube was allowed to stand for a few more days to ensure the completion of the phase separation. Volumes of the two phases were measured by graduations on the tube. Then a small amount of the solvent was added, the total amount was weighed again, and the procedure was repeated until the mixture was sufficiently diluted as to yield a uniform solution. When the phase separation had not taken place, the test was reversed, i.e., a small amount of the solvent was evaporated, the total amount was weighed, and the procedure was repeated until the mixture was sufficiently concentrated as to exhibit the phase separation. Repetition of these two procedures should result in a more accurate determination of the critical concentration. 


\section{H. O hNuma, T. KotAKA and H. INAGAKI}

\section{RESULTS*}

\section{Characterization of Polymer Samples}

Data of comonomer composition (by ST-weight fraction $\mathrm{x}$ or by ST-mole fraction $\mathrm{m}$ ), numberaverage molecular weight $M_{n}$, and weight-average molecular weight $M_{w}$ of the block copolymer samples used here are listed in Table I, together with the data of their precursor PST samples. ${ }^{2}$
The block copolymer samples are of the PMMAPST - PMMA type with nearly equimolar compositions. It should be noted that for a copolymer sample with compositional heterogeneity the light-scattering method ${ }^{6,23}$ and also the sedimentation equilibrium method ${ }^{22,24}$ merely allow one to determine apparent molecular weights, which depend on the solvent nature through the specific refractive increment and the

Table I. Characterization data of precursor PST samples and block copolymers of PMMA-PST-PMMA type. ${ }^{a}$

\begin{tabular}{|c|c|c|c|c|c|c|}
\hline \multicolumn{3}{|c|}{ Precursor PST $-\mathrm{H}$} & \multicolumn{4}{|c|}{ PMMA-PST_PMMA block copolymer-B } \\
\hline Code & $10^{-4} M_{n}$ & $10^{-4} M_{w}$ & Code & $x$ & $10^{-4} M_{n}$ & $10^{-4} M_{w}$ \\
\hline $29 \mathrm{H}$ & 0.985 & 1.78 & 29B & 0.53 & 1.96 & 3.16 \\
\hline $28 \mathrm{H}$ & 3.19 & 5.20 & $28 \mathrm{~B}$ & 0.47 & 6.62 & 9.19 \\
\hline $25 \mathrm{H}$ & 6.72 & 13.95 & $25 B$ & 0.49 & 13.5 & 26.0 \\
\hline $26 \mathrm{H}$ & 11.5 & 14.4 & 26B & 0.46 & 25.9 & 30.8 \\
\hline $22 \mathrm{H}$ & 26.8 & 38.0 & $22 B$ & 0.45 & 58.2 & 80.7 \\
\hline $23 \mathrm{H}$ & 62.0 & 74.6 & $23 \mathrm{~B}$ & 0.61 & 98.0 & 114.2 \\
\hline $27 \mathrm{H}$ & 54.8 & 67.1 & $27 \mathrm{~B}$ & 0.41 & 124.0 & 147.4 \\
\hline
\end{tabular}

a The $M_{n}$ were determined by osmometry in TOL at $30.0^{\circ} \mathrm{C}$; the $M_{w}$ of some copolymers were determined by the light-scattering method in different solvents such as MEK, TOL, and 1,2-dichloroethane. For details, see ref 2 . The sedimentation equilibrium method was applied to low molecular weight materials in MEK at $30.0^{\circ} \mathrm{C}$, whereas the light-scattering method was used for high-molecular weight materials.

Table II. Characterization data of PMMA samples

\begin{tabular}{ccc}
\hline Code & $10^{-4} M_{n}$ & $10^{-4} M_{w}$ \\
\hline $14 \mathrm{M}$ & 7.21 & 18.9 \\
$40 \mathrm{M}$ & 11.73 & 19.2 \\
$31 \mathrm{M}$ & 16.6 & 20.0 \\
$38 \mathrm{M}$ & 34.3 & 40.0 \\
$41 \mathrm{M}$ & 46.3 & 51.4 \\
$42 \mathrm{M}$ & 100 & 124 \\
\hline
\end{tabular}

a See footnote on Table I.

partial specific volume of the solute. Directly observed data have to be corrected for heterogeneity to determine the true $M_{w}$. The procedures are described elsewhere. ${ }^{2}$ Table II lists the molecular weight data of PMMA samples used here.

\section{Intrinsic Viscosity Data}

Measurements of viscosities of the block copolymers were made in several solvents which had been chosen because of the difference in

* Numerical data are available upon request. their behavior toward PST and PMMA. Cyclohexanol (CHL) has nearly equal $\theta$-temperatures toward all three polymers, PST $\left(82^{\circ} \mathrm{C}\right)$, PMMA $\left(79^{\circ} \mathrm{C}\right)$, and ST-MMA block copolymers $\left(81^{\circ} \mathrm{C}\right) .^{1,3,6,11 \mathrm{a}}$ Tetrahydrofuran (THF) is a good solvent for both PST and PMMA. Toluene (TOL) is a good solvent for PST and a moderate solvent for PMMA, whereas 2-butanone (MEK) is the opposite. Diethyl malonate (DEM) is a $\theta$ solvent for $\operatorname{PST}\left(35.9^{\circ} \mathrm{C}\right)^{25 a}$ and a moderate solvent for PMMA. Whereas 1-chloro-n-butane $\left(1-\mathrm{CB} ; 35.5^{\circ} \mathrm{C}\right)^{25 \mathrm{~b}}$ and $p$-xylene $\left(p\right.$-XY; $\left.\sim 35^{\circ} \mathrm{C}\right)$ are $\theta$ solvents for PMMA and moderate solvents for PST.

All the viscometric data were extrapolated to infinite dilution to estimate the intrinsic viscosity [ $\eta]$ and the Huggins constant $k^{\prime}$ by use of the following three equations:

$$
\begin{gathered}
\eta_{\mathrm{sp}} / c=[\eta]+k^{\prime}[\eta]^{2} c+\mathrm{O}\left(c^{2}\right) \\
\eta_{\mathrm{sp}} / c=[\eta]+k^{\prime}[\eta] \eta_{\mathrm{sp}}+\mathrm{O}\left[\left(\eta_{\mathrm{sp}}\right)^{2}\right] \\
\left(\ln \eta_{r}\right) / c=[\eta]-\left(0.5-k^{\prime}\right)[\eta]^{2} c+\mathrm{O}\left(c^{2}\right)
\end{gathered}
$$




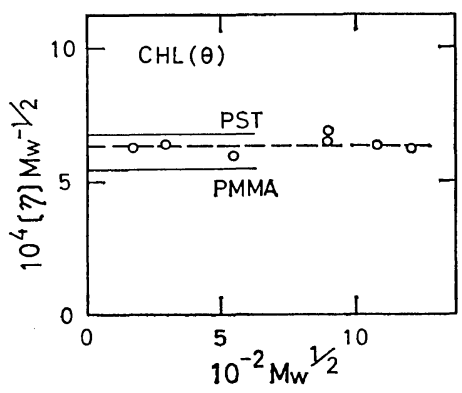

Figure 1. Plots of $[\eta] M_{w}^{-1 / 2}$ vs. $M_{w}^{1 / 2}$ for nearly equimolar block copolymers of PMMA-PSTPMMA type (circles), PST and PMMA (solid curves) in $\mathrm{CHL}$ at the respective $\theta$ temperatures (see text for details).

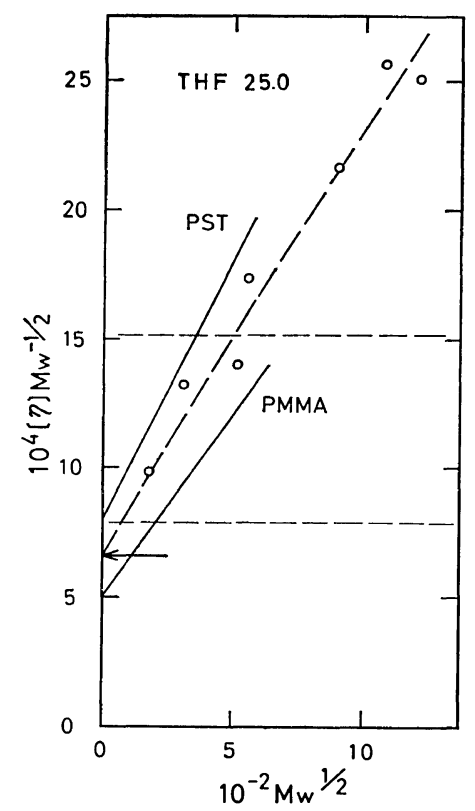

Figure 2. Plots of $[\eta] M_{w}{ }^{-1 / 2} v s . M_{w}^{1 / 2}$ for the same polymers, as in Figure 1, in a common solvent, $\mathrm{THF}$, at $25.0^{\circ} \mathrm{C}$. The arrow indicates the value of $K_{0}$ for the block copolymers as computed by eq $3 b, 5 a$ and $b$. The horizontal broken lines indicate the values of $\alpha_{\eta}^{3}=1.2$ and 2.3 [cf. eq 4a].

Here $\eta_{r}$ and $\eta_{\mathrm{sp}}$ are the relative and the specific viscosity, respectively, and $c$ is the solute concentration (in $\mathrm{g} / 100 \mathrm{ml}$ ). Value of $[\eta]$ of each system was determined by extrapolating these three types of plots so as to yield a common intercept at $c=0$. Values of $k^{\prime}$ thus deduced did not always exactly coincide. However we

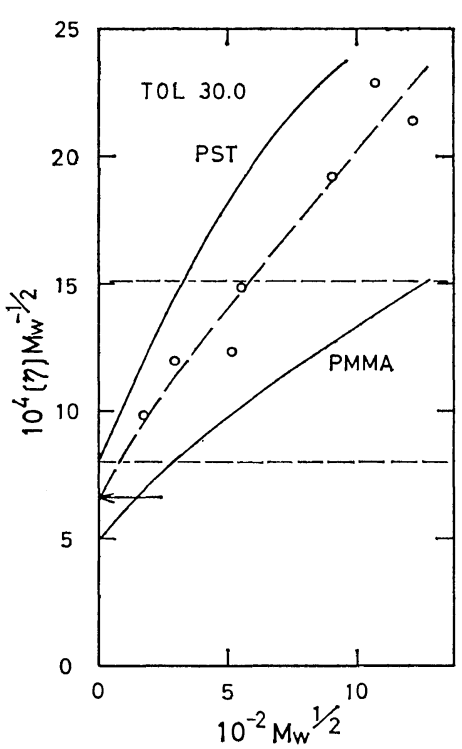

Figure 3. Plots of $[\eta] M_{w}{ }^{-1 / 2} v s . M_{w}^{1 / 2}$ for the same polymers as before in TOL at $30.0^{\circ} \mathrm{C}$. For symbols, see Figures 1 and 2.

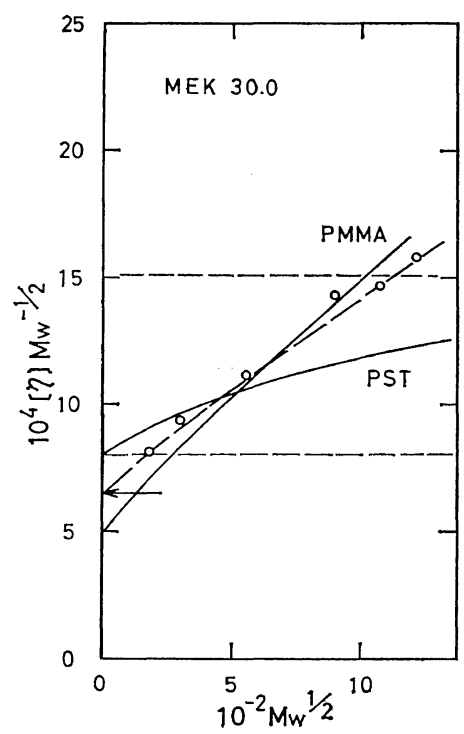

Figure 4. Plots of $[\eta] M_{w}^{-1 / 2} v s . M_{w}^{1 / 2}$ for the same polymers as before in MEK at $30.0^{\circ} \mathrm{C}$. For symbols, see Figures 1 and 2.

did not attempt to force them to fit by introducing an additional correlation among the expansion coefficients of higher order terms, as had been done by Berry. ${ }^{26}$ The $[\eta]$ data together 


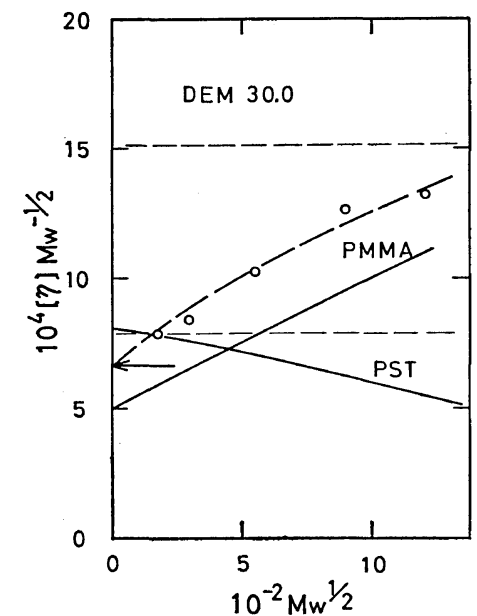

Figure 5. Plots of $[\gamma] M_{w}^{-1 / 2} v s . M_{w}^{1 / 2}$ for the same polymers as before in a selective $\theta$ solvent, DEM, at $30.0^{\circ} \mathrm{C}$. For symbols, see Figures 1 and 2.

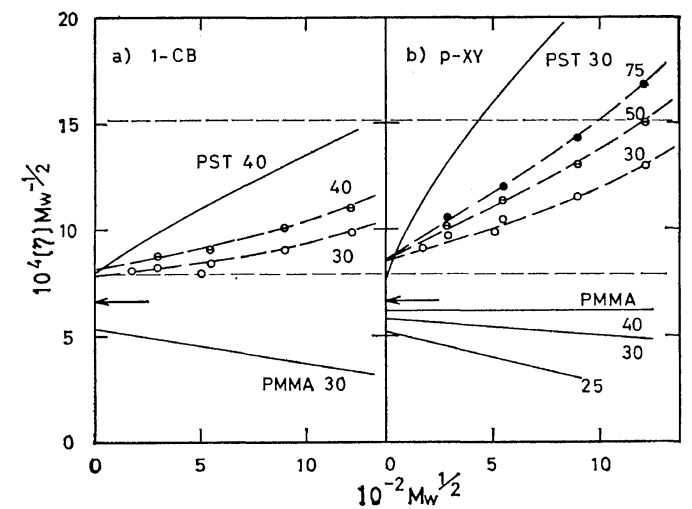

Figure 6. Plots of $[\eta] M_{w}^{-1 / 2} v s . M_{w^{1 / 2}}$ for the same polymers as before in selective $\theta$ solvents, (a) 1 $\mathrm{CB}$ and (b) $p$-XY, at temperatures as indicated. For symbols, see Figures 1 and 2.

with those of the parent homopolymers are shown in Figures $1-6$ plotted in the now familiar

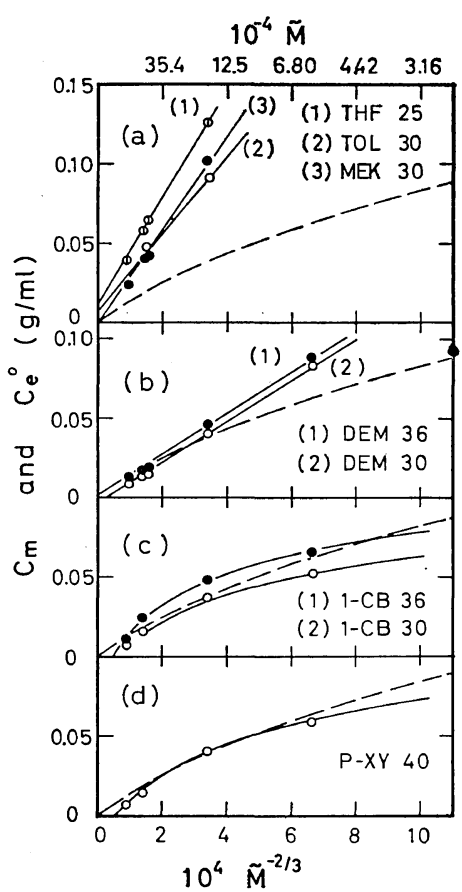

Figure 7. Plot of $c_{\mathrm{m}} v s . \widetilde{M}^{-2 / 3}$ for equimolar mixtures of PST and PMMA in various solvents and temperatures as indicated. Dashed curve in each figure is the relation of $c_{e}^{0} v s . \widetilde{M}^{-2 / 3}$ computed by eq $9 a$ and $b$. For details, see text.

form of $[\eta] M_{w}{ }^{-1 / 2}$ vs. $M_{w}{ }^{1 / 2}$ plot. $^{27}$

Phase Separation Test in Ternary Systems

The phase separation test was carried out on mixtures of PST and PMMA in the same solvents employed in the viscosity measurements. First the behavior of an equimolar blend of PST-26H and PMMA-14M was examined in $\mathrm{CHL}$ at $85^{\circ} \mathrm{C}$. We found that phase separation does not take place even up to a concentration as high as $w=0.20$ (in g-total polymer/ g-solution). We did not carry out the test any

Table III. Dependence of critical miscibility concentration on polymer composition in PST : PMMA : TOL system

\begin{tabular}{cccc}
\hline PST-wt fract & $c_{\mathrm{m}}, \mathrm{g} / \mathrm{m} l$ & PST-wt fract & $c_{\mathrm{m}}, \mathrm{g} / \mathrm{m} l$ \\
\hline PST-26H : PMMA-14M : TOL $30^{\circ} \mathrm{C}$ & PST-27H : PMMA-38M : TOL $30^{\circ} \mathrm{C}$ \\
0.2959 & 0.0941 & 0.1500 & 0.0545 \\
0.5002 & 0.0928 & 0.3038 & 0.0510 \\
0.7006 & 0.0940 & 0.4996 & 0.0489 \\
& & 0.6987 & 0.0500 \\
& & 0.8496 & 0.0526 \\
\hline
\end{tabular}


further, and could not locate the critical concentration $c_{\mathrm{m}}$ of the PST : PMMA : CHL systems. However, the result implies that CHL has a very high compatibilizing power toward the PST : PMMA pair.

The similar test was made in TOL at $30^{\circ} \mathrm{C}$. First the dependence of $c_{\mathrm{m}}$ (g-total polymer $/ \mathrm{m} l$ solution) on the mixing ratio was examined with two different pairs, i.e., PST-26H : PMMA-14M and PST-27H:PMMA-38M. The results are summarized in Table III. In each pair the dependence of $c_{\mathrm{m}}$ on the mixing ratio is very small and the critical concentration (the plait point) is found for the equimolar mixture. Apparently PST and PMMA are far less compatible in $\mathrm{TOL}\left(30^{\circ} \mathrm{C}\right)$ than in $\mathrm{CHL}\left(85^{\circ} \mathrm{C}\right)$. Further tests were conducted on several equimolar mixtures.

On the incompatibility in ternary systems, Berek, et al. ${ }^{28}$ reported that the critical concentration $c_{\mathrm{m}}$ decreases with increasing solute molecular weights according to the following empirical relation

$$
c_{\mathrm{m}}=C^{\prime} \tilde{M}^{-2 / 3}+C^{\prime \prime} ; \tilde{M}=\left(M_{\mathrm{A}} M_{\mathrm{B}}\right)^{1 / 2}(2 \mathrm{a}, \mathrm{b})
$$

Here $C^{\prime}$ and $C^{\prime \prime}$ are constants characteristic of a given ternary system, and $\tilde{M}$ is the geometric mean-molecular weight of the two polymers. As shown in Figure 7a, the PST : PMMA : TOL system is found to obey eq $2 \mathrm{a}$ and $\mathrm{b}$, and the $c_{\mathrm{m}}$ vs. $\tilde{M}^{-2 / 3}$ data are in close agreement with those reported by Berek, et al. ${ }^{28}$ on the same system. The test was also conducted on equimolar mixtures of PST and PMMA in THF $\left(25^{\circ} \mathrm{C}\right)$, MEK $\left(30^{\circ} \mathrm{C}\right)$, DEM $\left(30\right.$ and $\left.36^{\circ} \mathrm{C}\right)$, 1$\mathrm{CB}\left(30\right.$ and $\left.36^{\circ} \mathrm{C}\right)$, and $p$-XY $\left(40^{\circ} \mathrm{C}\right)$. The $c_{\mathrm{m}}$ vs. $\tilde{M}^{-2 / 3}$ data for these systems are shown in Figures 7a-7d. In three solvents-THF, MEK, and DEM - the data also obey eq $2 \mathrm{a}$ and $\mathrm{b}$ ( $c f$. Figures $7 \mathrm{a}$ and $\mathrm{b}$ ). However in two other solvents-1-CB and $p$-XY-eq $2 \mathrm{a}$ and $\mathrm{b}$ appear to be unsatisfactory ( $c f$. Figures $7 \mathrm{c}$ and $\mathrm{d}$ ). The reason for this difference is not clear at the moment. Apparently, the fact that the latter two solvents are $\theta$ solvents for one of the constituent homopolymers could not be the sole reason for the difference, since it has not been observed in DEM which also is a $\theta$ solvent for one of the constituents, PST. However the poor compatibility of the PST : PMMA pair in
Table IV. Values of parameters in critical miscibility concentration versus geometric meanmolecular weight relationship, $c_{\mathrm{m}}=C^{\prime} \tilde{M}^{-2 / 3}$ $+C^{\prime \prime}$, for equimolar mixtures of PST and PMMA in various solvents ${ }^{\mathrm{a}}$

\begin{tabular}{ccc}
\hline Solvent (temp, $\left.{ }^{\circ} \mathrm{C}\right)$ & $10^{-2} C^{\prime}$ & $C^{\prime \prime} \mathrm{g} / \mathrm{m} l$ \\
\hline THF (25) & 3.05 & 0.011 \\
TOL (30) & 2.46 & 0.005 \\
MEK (30) & 2.72 & $0.001_{5}$ \\
DEM (36) & 1.32 & 0.001 \\
$(30)$ & 1.30 & -0.002 \\
\hline
\end{tabular}

a Values of $\tilde{M}$ were computed from $M_{w}$ data; $c_{\mathrm{m}}$ (g-total polymer $/ \mathrm{m} l$-solution) were determined for the mixtures with $10^{-4} \tilde{M}=113.0-5.85$.

DEM, 1-CB, and $p-\mathrm{XY}$ may be attributed partly to the fact that they are selective $\theta$ solvents, i.e., those for either one of the constituent homopolymers. Table IV lists values of $C^{\prime}$ and $C^{\prime \prime}$ for the systems that have been found to obey eq $2 \mathrm{a}$ and $\mathrm{b}$. From all the above results we may conclude that the PST: PMMA pair becomes more and more incompatible in the order $\mathrm{CHL}\left(85^{\circ} \mathrm{C}\right) \ll \mathrm{THF}<\mathrm{TOL}<\mathrm{MEK}<\mathrm{DEM}$ $<1-\mathrm{CB} \cong p-\mathrm{XY}$.

\section{DISCUSSION}

\section{Estimation of Interaction Parameters}

For homopolymer solutions the short-range ${ }^{29}$ and the long-range (excluded volume) ${ }^{30}$ interaction parameters may be estimated experimentally by use of appropriate $[\eta] v s . M_{w}$ equations. ${ }^{26,27,31,32}$ The short-range interaction parameter may be computed from $[\eta]_{\theta}$ data by

$$
[\eta]_{\theta}=K_{0} M_{w}{ }^{1 / 2} ; K_{0}=\Phi_{0}\left[6\left\langle s^{2}\right\rangle_{0} / M_{w}\right]^{3 / 2}
$$

where $\Phi_{0}$ is the Flory-Fox viscosity constant ${ }^{30}$ under the $\theta$ condition $\left(10^{-21} \Phi_{0}=2.68\right),{ }^{33}\left\langle s^{2}\right\rangle_{0}$ is the unperturbed mean-square molecular radius, and the ratio $\left\langle s^{2}\right\rangle_{0} / M_{w}$ is a parameter representing the short-range interactions. ${ }^{27,29}$

The long-range interactions in good solvent systems may be determined from [ $\eta]$ vs. $M_{w}$ data by separating the effects of the short and the long-range interactions. ${ }^{27}$ For this purpose several $[\eta]$ equations have been proposed so far. ${ }^{26,27,31,32}$ Among them an equation recently proposed by Fujita, et al., ${ }^{32 \mathrm{a}}$ reads 


$$
\begin{gathered}
{[\eta] M_{w}{ }^{-1 / 2}=1.05 K_{0}+0.83 \Phi_{0} B M_{w}{ }^{1 / 2}} \\
\left(\text { for } 1.2<\alpha_{\eta}{ }^{3}=[\eta] /[\eta]_{\theta}<2.3\right)
\end{gathered}
$$

The parameter $B$ is proportional to the binary cluster integral $\beta$ for a pair of segments within a chain of molecular weight $M$ and consisting of $n$ such segments:

$$
B=(4 \pi)^{-3 / 2} \beta(n / M)^{2}
$$

Eq $4 \mathrm{a}$ was so constructed that values of $B$ estimated are made to agree with those from mean-square radius $\left\langle s^{2}\right\rangle$ data and from osmotic second virial coefficient $A_{2}$ data. $^{32}$ In that sense the equation is consistent with the framework of the two parameter theories ${ }^{34}$ that demand the identity between the interaction parameters of intrachain segment pairs (those relevant to $\left\langle\boldsymbol{s}^{2}\right\rangle$ and [ $\left.\eta\right]$ data) and of interchain segment pairs (those relevant to $A_{2}$ data).

Application of these equations to block copolymer data requires further considerations. One problem is concerned with the concept of the $\theta$ condition and the other with conformational anomalies which might prevail in certain particular solvents. Previously we referred to a condition wherein overall interchain excluded volume interactions vanish so that $A_{2}=0$ as the " $\theta$ " condition for a block copolymer solution. ${ }^{1,3,6}$ However we wish to emphasize that even under the " $\theta$ " condition all types of intrachain interactions between like and unlike segment pairs are unlikely to vanish simultaneously. ${ }^{1,3}$ Consequently eq $4 a$ and $b$ might not always be valid for block copolymers under the ' $\theta$ ' condition. In fact we found in 2-ethoxyethanol solutions of PMMA-PST-PMMA block copolymers that $[\eta]_{\theta}$ is not proportional to $M_{w}{ }^{1 / 2}$ even under the condition of $A_{2}$ being zero. $^{1,3}$

On the other hand, eq $4 \mathrm{a}$ or any other equations of similar nature might not always be applicable to block copolymer data for estimating $K_{0}$ and $B$ separately. Namely in certain special solvents the excluded-volume interactions between like and unlike segment pairs might induce conformational anomalies in block copolymer chains. In such an event eq $4 a$, which is essentially based on a random coil model with a uniform expansion approximation, would become invalid.
First we assume a hypothetical unperturbed state for block copolymer solution, wherein all types of long-range interactions vanish simultaneously. Then the unperturbed dimensions of a block copolymer chain may be readily correlated to those of its parent homopolymers. ${ }^{35,36}$ For a block copolymer chain of symmetric $\operatorname{poly}(\mathrm{B})-\operatorname{poly}(\mathrm{A})-\operatorname{poly}(\mathrm{B})$ type (BAB-type) in which two B-subchains have the same length, the $\left\langle s^{2}\right\rangle_{0} / M_{w}$ may be written as

$$
\begin{aligned}
\frac{\left\langle s^{2}\right\rangle_{0}}{M_{w}}= & x\left[\frac{\left\langle s^{2}\right\rangle_{0}}{M_{w}}\right]_{\mathrm{A}}+(1-x)\left[\frac{\left\langle s^{2}\right\rangle_{0}}{M_{w}}\right]_{\mathrm{B}} \\
& +\frac{x}{2}(1-x)(1+x) \Delta \\
\Delta & =\left[\frac{\left\langle s^{2}\right\rangle_{0}}{M_{w}}\right]_{\mathrm{A}}-\left[\frac{\left\langle s^{2}\right\rangle_{0}}{M_{w}}\right]_{\mathrm{B}}
\end{aligned}
$$

where $x$ is the composition in weight fraction of A (in this case ST), and the subscripts A and $B$ denote the quantities characteristic of the parent homopolymers. ${ }^{6,35}$

On the other hand application of the perturbation treatment $t^{37}$ to block copolymer solution leads to an equation of the parameter $\beta$ as

$$
B=p_{\mathrm{AA}} B_{\mathrm{AA}}+p_{\mathrm{BB}} B_{\mathrm{BB}}+2 p_{\mathrm{AB}} B_{\mathrm{AB}}
$$

Here $p_{i j}(i, j=\mathrm{A}, \mathrm{B})$ is the probability of the $i-j$ contacts and depends on the composition and the chain-architecture such as AB-type ${ }^{11}$ or BAB-type $^{36}$ of the block copolymer chain. The parameter $B_{i j}$ characterizes the $i-j$ interaction: The parameters $B_{\mathrm{AA}}$ and $B_{\mathrm{BB}}$ may be identified with those of the solutions of the respective parent homopolymers. Whereas the parameter $\boldsymbol{B}_{\mathrm{AB}}$ may be correlated with a similar quantity $\chi_{\mathrm{AB}}$, which characterizes the incompatible interactions in the ternary system, $\operatorname{poly}(\mathbf{A})$ : $\operatorname{poly}(\mathbf{B})$ : solvent: $:^{5,6}$

$$
\chi_{\mathrm{AB}} \propto \Delta B_{\mathrm{AB}}=B_{\mathrm{AB}}-\left(B_{\mathrm{AA}}+B_{\mathrm{BB}}\right) / 2
$$

In a fully perturbed state the parameter $B$ may be written in the form of eq $6 a^{5,38}$ as well. However a calculation of the $p_{i j}$ requires a knowledge of the segment distribution under the perturbed state. Instead of carrying out the calculation, we assume that the segment distribution of a block copolymer chain may be approximated, if not always, by a random distribution of disconnected segments. Then the 
probability becomes $p_{i j}=m_{i} m_{j}$ with $m_{i}\left(m_{\mathrm{A}}=m\right.$ and $\left.m_{\mathrm{B}}=1-m\right)$ being the mole fraction of $i$-segment (more strictly the volume fraction). ${ }^{5,638}$

$$
B=m^{2} B_{\mathrm{AA}}+(1-m)^{2} B_{\mathrm{BB}}+2 m(1-m) B_{\mathrm{AB}}(6 \mathrm{c})
$$

The assumption in eq $6 \mathrm{c}$ is that the blockcopolymer chain would not exhibit conformational anomalies. It should be then legitimate to introduce a further assumption that the viscosity equations, eq $4 \mathrm{a}$ and $6 \mathrm{c}$ would be valid for the block copolymer solution. On the other hand, if the block copolymer chains assume anomalous conformations, eq $6 \mathrm{c}$ should be invalid and its $[\eta]$ vs. $M_{w}$ relationship would also exhibit anomalies. In other words, when the $[\eta] M_{w}{ }^{-1 / 2} v s . M_{w}{ }^{1 / 2}$ relationship for a given block copolymer solution obeys eq $4 \mathrm{a}$ so that the plot allows a reasonable estimate of $K_{0}$, its slope should yield the parameter $B$ that can be given by eq $6 \mathrm{c}$.

The criterion mentioned above is apparently too crude, but may be employed as a working hypothesis. On this basis we examined the [ $\eta]$ data shown in Figures $1-6$. In each figure an arrow on the abscissa indicates the value of $K_{0}$ computed by eq $3 \mathrm{~b}, 5 \mathrm{a}$, and $5 \mathrm{~b}$ with appropriate values of $\left(\left\langle s^{2}\right\rangle_{0} / M_{w}\right)$ for PST and PMMA. ${ }^{6}$ As discussed previously, ${ }^{1,3}$ the behavior in CHL at $81^{\circ} \mathrm{C}$ ( $c f$. Figure 1) is especially noteworthy. Since the $\theta$ temperatures of PST and PMMA in $\mathrm{CHL}$ are both quite close to the values of " $\theta$ " of ST-MMA block copolymers, ${ }^{1,6,11}$ the values of $B_{\mathrm{AA}}$ and $B_{\mathrm{BB}}$ may be regarded as practically zero. Then the feature of the $[\eta]_{\theta}$ data, $i . e$. , their proportionality to $M_{w}{ }^{1 / 2}$ and the agreement between the experimental and computed values of $K_{0}$, suggests that the parameter $B$ of ST-MMA block copolymers should be zero also. The result may be interpreted by an alternative of either $p_{\mathrm{AB}}=\mathbf{0}$ or $B_{\mathrm{AB}}=0$. The former, $p_{\mathrm{AB}}=0$, means that the block copolymer chain assumes a particular conformation in which the domains of PST and PMMA-subchains are completely segregated, i.e., a segregated-chain model. Then one unavoidably encounters difficulty in explaining the feature of the $[\eta]_{\theta}$ data and also the fact that $A_{2}=0$ on the basis of the segregated-chain model. On the other hand the latter, $B_{\mathrm{AB}}=0$, well explains the above difficulty but leads to conflict with the currently accepted concept that the $B_{A B}$ is primarily governed by the nature of the polymer-polymer interactions and is positive for an incompatible pair such as PST : PMMA..$^{5,7-9}$ However, the phase separation test on the ternary system, PST : PMMA : CHL, has shown that, as opposed to this concept, the pair is highly compatible in CHL. Consequently the parameter $\chi_{\mathrm{AB}}$ and hence $B_{\mathrm{AB}}$ may be regarded as practically zero in this system. Judging from all this evidence, we are inclined to believe that the " $\theta$ " condition for ST-MMA block copolymer in CHL is an exceptionally genuine $\theta$ condition: all types of excluded-volume interactions vanish simultaneously and the block copolymer chains would assume a random flight conformation. ${ }^{1,3}$

Turning our attention to other systems, we find that $[\eta] M_{w}{ }^{-1 / 2} v s . M_{w}{ }^{1 / 2}$ plots in THF, TOL, MEK, and DEM yield reasonable estimates

Table V. Values of long-range interaction parameters for PST, PMMA, and nearly equimolar block copolymers of PMMA-PST-MMA type in various solvents

\begin{tabular}{lcccc}
\hline \multirow{2}{*}{ Solvent $\left(\right.$ temp, $\left.{ }^{\circ} \mathrm{C}\right)$} & PST, $10^{-30} B_{\mathrm{AA}}$ & PMMA, $10^{-30} B_{\mathrm{BB}}$ & \multicolumn{2}{c}{ PMMA-PST-PMM } \\
\cline { 4 - 5 } & & & $10^{-30} B$ & $10^{-30} \Delta B_{\mathrm{AB}^{\mathrm{b}}}$ \\
\hline CHL (81) & 0.0 & 1.0 & 0.0 & -1.0 \\
THF (25) & 61.5 & 42.1 & 52.4 & 1.2 \\
TOL & 62.5 & 23.8 & 45.2 & 4.0 \\
MEK & 10.7 & 28.3 & 25.6 & -2.2 \\
DEM & -6.5 & 14.9 & 18.5 & - \\
1-CB & 13.7 & -4.2 & - & \\
$p$-XY & 53.6 & -6.0 & & \\
\hline a Data obtained at $30.0^{\circ} \mathrm{C}$ unless otherwise noted. & & \\
b Computed by eq $6 \mathrm{~b}$ and c. & &
\end{tabular}




\section{H. Ohnuma, T. Kotaka and H. Inagaki}

of $K_{0}$, which are in close agreement with the computed value ( $c f$. Figures 2, 3, 4, and 5). The plots in these solvents appear to obey eq 4a. Hence we have estimated the values of $B$ by eq $4 \mathrm{a}$, from which we further compute the values of $\Delta B_{\mathrm{AB}}$ by eq $6 \mathrm{~b}$ and $\mathrm{c}$. The results are listed in Table $\mathrm{V}$. Interestingly the value of $\Delta B_{\mathrm{AB}}$ increases in the order $\mathrm{CHL}\left(81^{\circ} \mathrm{C}\right)$ $<$ THF $<$ TOL $<$ MEK $<$ DEM. The tendency corresponds to the tendency of increasing incompatibility in these solvents.

As demonstrated in Figures $6 \mathrm{a}$ and $\mathrm{b}$, the plots of $[\eta] M_{w}{ }^{-1 / 2}$ vs. $M_{w}^{1 / 2}$ exhibit significant anomalies in 1-CB and $p$-XY. In both solvents, the extrapolation to $M_{w}^{1 / 2}=0$ yields unduly large values of $K_{0}$ in comparison with the computed value. Moreover, the plots exhibit a somewhat upward concavity that is just the opposite to the behavior usually encountered. It is noticeable that these anomalies persist at elavated temperatures significantly higher than the $\theta$ temperatures for PMMA. For such data eq $4 \mathrm{a}$ would not be applicable.

These anomalies should be attributed, at least partly, to the poor compatibilizing nature of the solvent involved that, in turn, may have resulted from the fact that it is a $\theta$ solvent for one of the constituent homopolymers. However it should be remembered that DEM is also a $\theta$ solvent for one of the constituents, PST, although no anomalies have been recognized in this solvent. Thus the differences in the behavior in DEM and in 1-CB and $p-\mathrm{XY}$ must be responsible for the chain architecture, i.e., to the fact that the block copolymers examined are of the PMMA-PST_PMMA type. Further discussion of this ploblem will take place in the following section.

\section{Possibility of Intrachain Phase Separation}

From the $[\eta]$ data for the block copolymer solutions and the phase separation test on the corresponding ternary systems, we may conclude that there is at least a qualitative correlation between the excluded volume interactions within unlike subchains of a block copolymer and the incompatibility of its parent homopolymer pair. Now the question to be asked is whether or not the phenomenon of intrachain phase separation is actually taking place in a block copolymer chain at infinite dilution. And if it has taken place, what conformation would the block copolymer chain assume?

To examine these points, we take the rather optimistic point of view. It would be legitimate to assume that the phenomenon would take place, if the effective concentration $c_{\mathrm{e}}$ of segments within the domain of an isolated block copolymer chain exceeds the critical miscibility concentration $c_{\mathrm{m}}$ of the corresponding ternary system. $^{3}$ On the other hand the intrachain phase separation would not take place if the $c_{\mathrm{e}}$ is lower than the $c_{\mathrm{m}}$. The latter part of the assumption might sound too crude and too optimistic. However we may, at least, expect that this would be the case.

To estimate the value of $c_{\mathrm{e}}$ for a block copolymer chain with molecular weight $M$, we replace it by a sphere with radius $\left\langle s^{2}\right\rangle^{1 / 2}$, and obtain

$$
c_{\mathrm{e}}=(3 / 4 \pi)\left(M / N_{\mathrm{a}}\left\langle s^{2}\right\rangle^{3 / 2}\right)=c_{\mathrm{e}}^{0} / \alpha^{3}
$$

where $N_{\mathrm{a}}$ is the Avogadro number, $c_{\mathrm{e}}^{0}$ is the effective-segment concentration for the chain under a hypothetical unperturbed state, and $\alpha^{2}=\left\langle s^{2}\right\rangle /\left\langle s^{2}\right\rangle_{0}$ is the square of the expansion factor due to the excluded volume interactions. The factor $\alpha^{2}$ is a single-valued, monotonically increasing function of an excluded volume variable $z=\left(\left\langle s^{2}\right\rangle_{0} / M\right)^{-3} B M^{1 / 2}$ and approaches unity in the limit of $z$ being zero (the unperturbed state). ${ }^{34}$ Therefore in any good solvent systems the $c_{\mathrm{e}}$ must be smaller than the $c_{\mathrm{e}}^{0}$. On the other hand the geometric mean-molecular weight $\tilde{M}$ of the chemically different subchains may be written for a triblock copolymer of symmetric BAB type with composition $x$ (in Aweight fraction) as

$$
\tilde{M}=[x(1-x) / 2]^{1 / 2} M
$$

Using the empirical value of $10^{18}\left\langle s^{2}\right\rangle_{0} / M_{w}=6.35$ obtained from $[\eta]_{\theta}$ data of the ST-MMA block copolymer-CHL solutions at $81^{\circ} \mathrm{C}$, and assuming the block copolymers being monodisperse, i.e., $M_{w}=M$ for the present system, we obtain

$$
c_{\mathrm{e}}^{0}=24.8 M^{-1 / 2} ; \tilde{M} / 2.828
$$

From these equations we can construct the $c_{\mathrm{e}}^{0}$ vs. $\tilde{M}^{-2 / 3}$ relation, which in turn may be compared with the $c_{\mathrm{m}}$ vs. $\tilde{M}^{-2 / 3}$ relationships for 
Table VI. Comparison of intrinsic viscosities of nearly equimolar block copolymers of PMMA-PSTPMMA type and of their precursor PST in p-xylene at $30.0^{\circ} \mathrm{C}$

\begin{tabular}{ccccccc}
\hline \multirow{2}{*}{ Code } & \multicolumn{2}{c}{ Precursor PST } & & \multicolumn{2}{c}{ PMMA-PST-PMMA } \\
\cline { 2 - 5 } & $10^{-4} M_{w}$ & {$[\eta], 100 \mathrm{~m} l / \mathrm{g}$} & & & & \\
& & & & & $10^{-4} M_{w}$ & {$[\eta], 100 \mathrm{~m} l / \mathrm{g}$} \\
\hline $28 \mathrm{H}$ & 5.20 & 0.273 & $28 \mathrm{~B}$ & 9.19 & 0.289 \\
$26 \mathrm{H}$ & 13.6 & 0.556 & $26 \mathrm{~B}$ & 30.8 & 0.582 \\
$22 \mathrm{H}$ & 38.0 & 1.090 & $22 \mathrm{~B}$ & 80.7 & 1.033 \\
$27 \mathrm{H}$ & 67.1 & 1.620 & $27 \mathrm{~B}$ & 147.4 & 1.590 \\
\hline
\end{tabular}

the ternary systems such as shown in Figures $7 \mathrm{a}-\mathrm{d}$.

It can be seen that in a group of solvents such as $\mathrm{CHL}\left(85^{\circ} \mathrm{C}\right)$, THF, TOL, and MEK, the $c_{\mathrm{m}}$ is much higher than the $c_{\mathrm{e}}^{0}$ and hence the $c_{\mathrm{e}}$ at all values of $\tilde{M}$, whereas in DEM the two concentrations become comparable in the region of large $\tilde{M}$, and similarly in 1-CB and $p$-XY they become comparable in almost the entire region of $\tilde{M}$. Judging from these results, we may say that ST-MA block copolymers would behave just as ordinary random coils in the former group of solvents, CHL, THF, TOL, and MEK. The $[\eta] v s$. $M_{w}$ data may be satisfactorily described by eq 4 a with a modification of the parameter $K_{0}$ and $B$ by eq $5 \mathrm{a}, \mathrm{b}$ and $6 \mathrm{c}$, respectively. The phenomenon of intrachain phase separation is quite unlikely to be taking place in such solvents.

In the latter group of solvents, DEM, 1-CB and $p-\mathrm{XY}$, the fact that these solvents are $\theta$ solvents for either one of the parent homopolymers appears to be important. The corresponding subchains might collapse in its $\theta$ solvent, and this might induce the intrachain phase separation, resulting in conformational anomalies. However the $[\eta]$ data in DEM appear to show no anomalies at all, or at least those detectable by $[\eta]$ measurements. The result may be interpreted as that in this solvent, DEM, the conformational change takes place at the central PST-subchains, whereas the side PMMA subchains behave normally. Such a change may not result in easily detectable anomalies in the

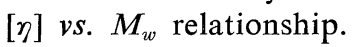

The situation must be entirely different in 1-CB and $p$-XY, since each block copolymer chain possesses two PMMA side chains flanking a PST-subchain. In fact significant anomalies were observed in the $[\eta]$ vs. $M_{w}$ relationships such as shown in Figure 6a and b. In this type of $\theta$ solvent, the two PMMA side chains presumably tend to collapse to form a single droplet within a block copolymer chain. In other words, they tend to undergo an intrachain association, thus severely restricting allowable conformations of the central PST subchain. In addition, the poor compatibility of PST and PMMA in these solvents may enhance conformational anomalies. The phenomenon is quite evident in the $[\eta]$ data obtained in $p$-XY at $30^{\circ} \mathrm{C}$. Table VI lists the $[\eta]$ data of several equimolar PMMA-PST_PMMA block copolymers together with those of their precursor PST. Surprisingly we notice that for high molecular weight samples the $[\eta]$ of a whole block copolymer chain is smaller than that of the precursor PST, which possesses only about half the molecular weight or chain length of the whole block copolymer chain. The anomaly must be attributed to the phenomenon of intrachain association of two PMMA-side chains within a block copolymer chain, just as mentioned above. If such is the case, the behavior of diblock copolymers of PST-PMMA type in the $\theta$ or nonsolvents toward PMMA must be different from those of triblock copolymers of the PMMAPST-PMMA type. In fact, our preliminary [ $\eta$ ] data on PST-PMMA diblock copolymers in $p$-XY and other nonsolvents toward PMMA show no anomalies of this nature, but rather suggest the formation of intermolecular aggregates. $^{39}$

Acknowledgement. One of us (H. O.) wishes to thank the Japan Society for the Promotion of Science for a post-doctorate fellowship from April 1968 through March 1969, which enabled 


\section{H. Ohnuma, T. Kotaka and H. InAgaki}

him to participate in this work.

\section{REFERENCES}

1. T. Kotaka, H. Ohnuma, and Y. Murakami, J. Phys. Chem., 70, 4099 (1966).

2. H. Ohmura, T. Kotaka, and H. Inagaki, Polymer, 10, 501 (1969).

3. T. Kotaka, H. Ohnuma, and H. Inagaki, ibid., 10, 517 (1969).

4. H. Ohnuma, Dissertation, Kyoto University, Kyoto, 1969.

5. W.H. Stockmayer, L.D. Moore, Jr., M. Fixman, and B. N. Epstein, J. Polym. Sci., 16, 517 (1955).

6. T. Kotaka, T. Tanaka, H. Ohnuma, Y. Murakami, and H. Inagaki, Polymer J., 1, 245 (1970).

7. A. Dobry and F. Boyer-Kawenoki, J. Polym. Sci., 2, 90 (1947).

8. R. L. Scott, J. Chem. Phys., 17, 268 (1949).

9. R. J. Kern and R. J. Solocombe, J. Polym. Sci., 15, 183 (1955).

10. (a) G. M. Burnett, D. Meares, and C. Paton, Trans. Faraday Soc., 58, 737 (1962); (b) S. Krause, J. Phys. Chem., 68, 1948 (1964); (c) J. R. Urwin and J. M. Stearne, Makromol. Chem., 78, 197, 204 (1964); Eur. Polym. J., 1, 227 (1965); (d) H. Inagaki, Makromol. Chem., 86, 289 (1965); H. Inagaki and T. Miyamoto, ibid., 87, 166 (1965).

11. (a) D. Froelich and H. Benoit, ibid., 92, 224 (1966); (b) D. Froelich, J. Chim. Phys., 64, 1311 (1968).

12. See, for example, T. Inoue, T. Soen, H. Kawai, M. Fukatsu, and M. Kurata, J. Polym. Sci., Part B, 6, 75 (1968).

13. See, for example, S. Krause, Macromolecules, 3, 84 (1970).

14. (a) M. Szwarc and A. Rembaum, J. Polym. Sci., 22, 189 (1956); (b) M. Szwarc, Fortschr. Hochpolym. Forsch., 2, 275 (1960).

15. M. Baer, J. Polym. Sci., Part A, 2, 417 (1964).

16. D. Freyss, P. Rempp, and H. Benoit, ibid., Part B, 2, 217 (1964).

17. T. Miyamoto and H. Inagaki, Polymer, J., 1, 46 (1970).

18. See, for example, "Organic Solvents," J. A. Riddick, and E. E. Toops, Jr., Ed., Interscienc Publishers Inc., New York, N.Y., 1955.

19. H. Inagaki, H. Suzuki, M. Fujii, and T. Matsuo, J. Phys. Chem., 70, 1718 (1966).

20. T. Kotaka, Y. Murakami, and H. Inagaki, ibid., 72, 829 (1968).

21. T. Kotaka and N. Donkai, J. Polym. Sci.,
Part A2, 6, 1745 (1968).

22. T. Kotaka, N. Donkai, and H. Inagaki, ibid., submitted.

23. (a) W. Bushuk and H. Benoit, Compt. Rend., 244, 3167 (1958); (b) H. Benoit and C. Wippler, J. Chim. Phys., 57, 524 (1960).

24. See, for example, R. L. Baldwin and K.E. van Holde, Fortschr. Hochpolym. Forsch., 1451 (1960).

25. (a) T. A. Orofino, J. Chem. Phys., 45, 4310 (1966); (b) G. V. Schulz, A. Haug, and R. Kirste, Z. Phys. Chem. (NF), 38, 1 (1963).

26. G. C. Berry, J. Chem. Phys., 44, 4550 (1966); ibid., 46, 1338 (1967).

27. (a) M. Kurata and W. H. Stockmayer, Fortschr. Hochpolym. Forsch., 3, 196 (1963); (b) W. H. Stockmayer and M. Fixman, J. Polym. Sci., Part C, 1, 137 (1963).

28. D. Berek, B. Bohmer, and D. Lath, Plaste u. Kautschuk, 14, 556 (1967).

29. See, for example, P. J. Flory, "Statistical Mechanics of Chain Molecules," Interscience Publishers, New York, N.Y., 1969.

30. P. J. Flory, "Principles of Polymer Chemistry," Cornell University Press, Ithaca, N.Y., 1953.

31. See, for example, (a) H. Inagaki, H. Suzuki, and M. Kurata, J. Polym. Sci., Part C, 15, 409 (1966); (b) J. M. G. Cowie, Polymer, 7, 387 (1966).

32. (a) T. Norisuye, K. Kawahara, A. Teramoto, and H. Fujita, J. Chem. Phys., 49, 4330 (1968); (b) K. Kawahara, T. Norisue, and H. Fujita, ibid., 49, 4339 (1968); (c) G. Tanaka, S. Imai, and H. Yamakawa, ibid., 52, 2639 (1970).

33. C. W. Pyunn and M. Fixman, ibib., 42, 3838 (1965).

34. W. H. Stockmayer, Makromol. Chem., 35, 54 (1960).

35. Th. G. Scholte, J. Polym. Sci., Part A-2, 5, 461 (1967).

36. T. Tanaka and T. Kotaka, unpublished.

37. (a) E. Teramoto, Busseiron Kenkyu, 39, 1 (1951); ibid., 40, 18 (1951); ibid., 41, 14 (1951); ibid., 44, 36 (1951); (b) B. H. Zimm, W. H. Stockmayer, and M. Fixman, J. Chem. Phys., 21, 1716 (1953); (c) M. Fixman, ibid., 23, 1656 (1955).

38. (a) L. A. Utracki, R. Simha, and L. J. Fetters, J. Polym. Sci., Part A-2, 6, 2051 (1968); (b) L. A. Utracki and R. Simha, Macromolecules, 1, 505 (1968).

39. T. Tanaka and T. Kotaka, unpublished experiments. 